\title{
Is supernova SN 2020faa an iPTF14hls look-alike?^
}

\author{
S. Yang (杨圣 $)^{1}$, J. Sollerman ${ }^{1}$, T.-W. Chen ${ }^{1}$, E. C. Kool ${ }^{1}$, R. Lunnan ${ }^{1}$, S. Schulze ${ }^{2}$, N. Strotjohann ${ }^{2}$, A. Horesh ${ }^{3}$, \\ M. Kasliwal ${ }^{4}$, T. Kupfer ${ }^{5}$, A. A. Mahabal ${ }^{6,7}$, F. J. Masci ${ }^{8}$, P. Nugent ${ }^{9,10}$, D. A. Perley ${ }^{11}$, R. Riddle ${ }^{4}$,
}

B. Rusholme ${ }^{8}$, and Y. Sharma ${ }^{4}$

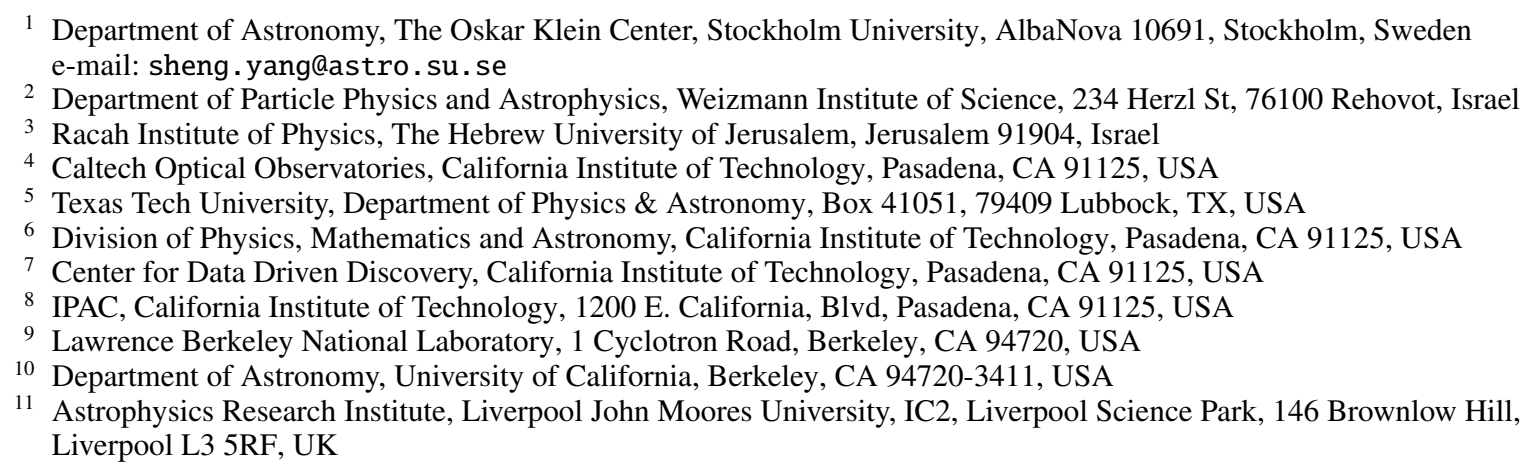

Received 15 September 2020 / Accepted 2 November 2020

ABSTRACT

\begin{abstract}
Context. We present observations of ZTF20aatqesi (SN 2020faa). This Type II supernova (SN) displays a luminous light curve (LC) that started to rebrighten from an initial decline. We investigate this in relation to the famous SN iPTF14hls, which received a great deal of attention and multiple interpretations in the literature, but whose nature and source of energy still remain unknown.

Aims. We demonstrate the great similarity between SN 2020faa and iPTF14hls during the first 6 months, and use this comparison to forecast the evolution of SN 2020faa and to reflect on the less well observed early evolution of iPTF14hls.

Methods. We present and analyse our observational data, consisting mainly of optical LCs from the Zwicky Transient Facility in the gri bands and of a sequence of optical spectra. We construct colour curves and a bolometric lc, and we compare ejecta-velocity and black-body radius evolutions for the two supernovae ( $\mathrm{SNe}$ ) and for more typical Type II SNe.

Results. The LCs show a great similarity with those of iPTF14hls over the first 6 months in luminosity, timescale, and colour. In addition, the spectral evolution of SN 2020faa is that of a Type II SN, although it probes earlier epochs than those available for iPTF14hls.

Conclusions. The similar LC behaviour is suggestive of SN 2020faa being a new iPTF14hls. We present these observations now to advocate follow-up observations, since most of the more striking evolution of SN iPTF14hls came later, with LC undulations and a spectacular longevity. On the other hand, for SN 2020faa we have better constraints on the explosion epoch than we had for iPTF14hls, and we have been able to spectroscopically monitor it from earlier phases than was done for the more famous sibling.
\end{abstract}

Key words. supernovae: general - supernovae: individual: SN 2020faa - supernovae: individual: ZTF20aatqesi supernovae: individual: iPTF14hls

\section{Introduction}

The extraordinary supernova (SN) iPTF14hls was a Type II SN, first reported by Arcavi et al. (2017, hereafter A17) as having a long-lived $(600+\mathrm{d})$ and luminous light curve (LC) showing at least five episodes of rebrightening. Sollerman et al. (2019, hereafter S19) followed the SN for 1000 days when it finally faded from visibility.

The spectra of iPTF14hls were similar to those of other hydrogen-rich SNe (i.e. dominated by Balmer lines with P Cygni profiles), but they evolved at a slower pace. A17 described a scenario where this could be the explosion of a very massive star that ejected a huge amount of mass prior to explosion. They connect such eruptions with the pulsational pair-instability mechanism.

\footnotetext{
* Photometric data are only available at the CDS via anonymous ftp to cdsarc.u-strasbg. fr (130.79.128.5) or via http://cdsarc. u-strasbg.fr/viz-bin/cat/J/A+A/646/A22
}

Following the report of A17 a large number of interpretations were suggested for this unusual object, which covered a wide range of progenitors and powering mechanisms. For example, Chugai (2018) agreed on the massive ejection scenario, while Andrews \& Smith (2018) argued for interaction with the circumstellar medium (CSM) as the source for the multiple rebrightenings in the LC, which was supported by S19. Dessart (2018) instead suggested a magnetar as the powering mechanism, whereas Soker \& Gilkis (2017) advocated a commonenvelope jet. Wang et al. (2018) proposed a fall-back accretion model for iPTF14hls, and Woosley (2018) discuss the pros and cons of several of these models, and whether the event was indeed a final explosion. Moriya et al. (2019) interpret the phenomenon as being due to a wind from a very massive star.

Taken together, this suite of publications demonstrate how extreme objects like iPTF14hls challenge most theoretical models and force us to expand the frameworks for transient phenomena. But iPTF14hls was a single specimen, until now. 
In this paper we present observations of SN 2020faa, a Type II SN that observationally appears to be similar to iPTF14hls during the first six months. We present gri LCs and optical spectra to highlight this similarity, and add information that was not available for iPTF14hls, such as earlier spectroscopy and better constraints on the explosion epoch. In addition to the groundbased observations, we obtained several epochs of data with the Neil Gehrels Swift Observatory (Swift, Gehrels et al. 2004). The main aim of this paper is to direct the attention of the community to this active transient, which may or may not evolve in the same extraordinary way as iPTF14hls did. If SN 2020faa becomes another iPTF14hls, we hope these observations, especially those at the early phases, will be complementary ingredients to iPTF14hls for the community to understand the physics of such peculiar long-lived transients.

The paper is structured as follows. In Sect. 2 we outline the observations and corresponding data reductions, including Sect. 2.1 where we present the detection and classification of SN 2020faa; the ground-based optical SN imaging observations and data reductions are presented in Sect. 2.2; in Sect. 2.3 we describe the Swift observations; a search for a precursor is done in Sect. 2.4; the optical spectroscopic follow-up campaign is presented in Sect. 2.5; and a discussion of the host galaxy is provided in Sect. 2.6. An analysis and discussion of the results are given in Sect. 3, which are summarised in Sect. 4.

For SN 2020faa, we adopt a redshift of $z=0.04106$ (see below), corresponding to a luminosity distance of $187 \mathrm{Mpc}$ (distance modulus $36.36 \mathrm{mag}$ ) using the same cosmology as A17. The Milky Way (MW) extinction is $E(B-V)=0.022 \mathrm{mag}$, and we adopt no host-galaxy extinction. We use the first ATLAS detection date as reference epoch for the phases. For iPTF14hls, we follow A17 and adopt a redshift of $z=0.0344$, corresponding to a luminosity distance of $156 \mathrm{Mpc}$. We correct the photometry for the MW extinction, $E(B-V)=0.014 \mathrm{mag}$, but make no correction for host-galaxy extinction. We follow A17 and use the PTF discovery date as a reference epoch for all phases for iPTF14hls.

\section{Observations and data reduction}

\subsection{Detection and classification}

The first detection of SN 2020faa (also known as ZTF20aatqesi) with the Palomar Schmidt 48-inch (P48) Samuel Oschin telescope was performed on 2020 March $28(\mathrm{JD}=2458936.8005)$ as part of the Zwicky Transient Facility (ZTF) survey (Bellm et al. 2019; Graham et al. 2019). The object had then already been discovered and reported to the Transient Name Server $\left(\mathrm{TNS}^{1}\right)$ by the ATLAS collaboration (Tonry et al. 2020) with a discovery date of March $24,2020\left(\mathrm{JD}_{\text {discovery }}=2458933.104\right)$ at $18.28 \mathrm{mag}$ in the cyan band, and a reported last non-detection (>18.57) 14 days before discovery in the orange band.

The first ZTF detection was made in the $g$ band, with a hostsubtracted magnitude of $18.40 \pm 0.09 \mathrm{mag}$, at the J2000.0 coordinates $\alpha=14^{\mathrm{h}} 47^{\mathrm{m}} 09.50^{\mathrm{s}}, \delta=+72^{\circ} 44^{\prime} 11.5^{\prime \prime}$. The first $r$-band detection came in $3.6 \mathrm{~h}$ later at $18.50 \pm 0.10$. The non-detections from ZTF include a $g$-band non-detection from 15 days before discovery, but this is a shallow global limit $(>17.46)$, whereas the one at 17 days before discovery is deeper at $>19.37 \mathrm{mag}$. The constraints on the time of explosion for SN 2020faa are thus not very precise, but in comparison with the very large uncertainty for iPTF14hls ( $\sim 100$ days) they are quite useful.

SN 2020faa is positioned on the edge of spiral galaxy WISEA J144709.05+724415.5, which did not have a reported

\footnotetext{
1 https://wis-tns.weizmann.ac.il
}

redshift in the $\mathrm{NED}^{2}$ catalogue, although the CLU catalogue (Cook et al. 2019) has it listed as CLU J144709.1+724414 at the same redshift as our spectroscopy provides. The SN together with the host galaxy and the field of view is shown in Fig. 1.

SN 2020faa was classified as a Type II SN (Perley et al. 2020) based on a spectrum obtained on 2020 April 6 with the Liverpool telescope (LT) equipped with the SPectrograph for the Rapid Acquisition of Transients (SPRAT) instrument. That spectrum revealed broad $\mathrm{H} \alpha$ and $\mathrm{H} \beta$ in emission, the blue edge being shifted by $\sim 9000 \mathrm{~km} \mathrm{~s}^{-1}$ with respect to the narrow emission line from the galaxy that provided the redshift $z=0.041$ consistent with CLU as mentioned above. The LT spectrum confirmed the tentative redshift and classification deduced from our first spectrum, obtained with the Palomar 60-inch telescope (P60; Cenko et al. 2006) equipped with the Spectral Energy Distribution Machine (SEDM; Blagorodnova et al. 2018). That first spectrum had already been taken on March 31, but the quality was not good enough to warrant a secure classification.

\subsection{Optical photometry}

Following the discovery we obtained regular follow-up photometry during the slowly declining phase in $g, r$, and $i$ bands with the ZTF camera (Dekany et al. 2020) on the P48 telescope. This first decline lasted for $\sim 50$ days, and no further attention was given to the $\mathrm{SN}$ during this time.

Later on, after rebrightening started, we also obtained a few epochs of triggered photometry in gri with the SEDM on the P60. The LCs from the P48 telescope come from the ZTF pipeline (Masci et al. 2019). Photometry from the P60 telescope were produced with the image-subtraction pipeline described in Fremling et al. (2016), with template images from the Sloan Digital Sky Survey (SDSS; Ahn et al. 2014). This pipeline produces PSF magnitudes, calibrated against SDSS stars in the field. All magnitudes are reported in the AB system.

The reddening corrections are applied using the Cardelli et al. (1989) extinction law with $R_{V}=3.1$. No further host-galaxy extinction has been applied since there is no sign of any Na I D absorption in our spectra. The LCs are shown in Fig. 2.

After the initial decline of about 50 days (which is past discovery in the observer's frame), SN 2020faa started to slowly brighten again. This continued for about 70 days and happened in all three bands. Once this was realised, in late May 2020, a more intense follow-up could be activated, in particular with regular spectroscopic observations (Sect. 2.5).

We used a Gaussian Processing (GP) algorithm ${ }^{3}$ to interpolate the photometric measurements and found that the peak happened at $m_{r}^{\text {peak }}=17.49 \pm 0.01$ after $t_{r}^{\text {rise }}=114.51 \pm 0.10$ rest frame days, via scipy.find_peaks. In the $g$ and $i$ bands the photometric behaviour follows the same trend, and peaked at $m_{g}^{\text {peak }}=17.83$ after $t_{g}^{\text {rise }}=114.70$ days as well as $m_{i}^{\text {peak }}=17.58$ after $t_{i}^{\text {rise }}=119.70$ rest frame days.

\subsection{Swift observations}

\subsubsection{UVOT photometry}

A series of ultraviolet (UV) and optical photometry observations were obtained with the UV Optical Telescope (UVOT) on board the Swift observatory (Gehrels et al. 2004; Roming et al. 2005).

\footnotetext{
2 https://ned.ipac.caltech.edu

3 https://george.readthedocs.io
} 

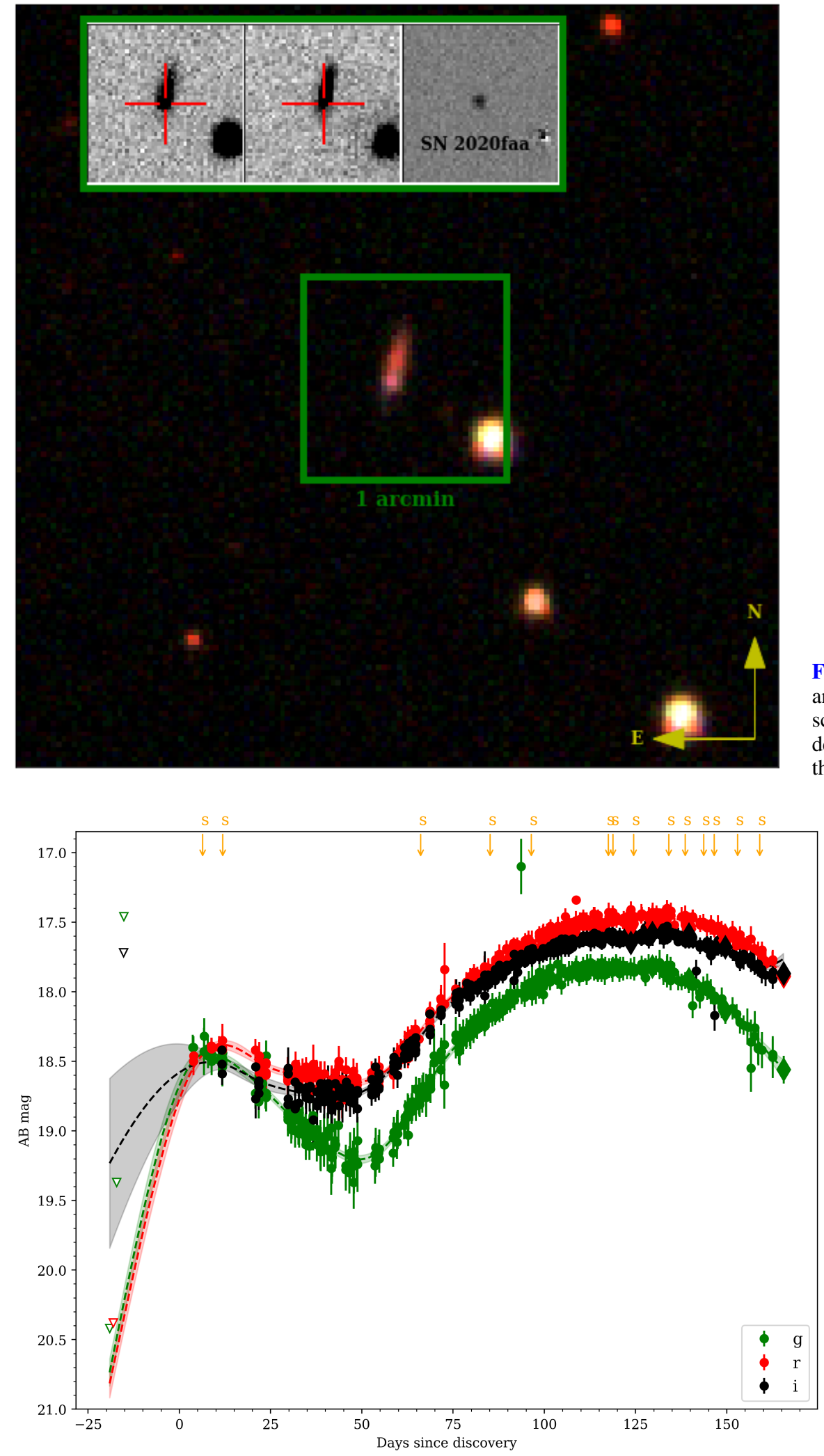

Fig. 1. A gri-colour composite image of SN $2020 \mathrm{faa}$ and its environment, as observed with the P48 telescope on 2020 April 5, eight days after the first ZTF detection. The $g$-band image subtraction is shown in the top panel.
Fig. 2. Light curves of SN 2020faa in $g$ band (green), $r$ band (red), and $i$ band (black). Shown are observed $(\mathrm{AB})$ magnitudes plotted vs the observer frame time in days since discovery. The yellow downward pointing arrows at the top indicate the epochs of spectroscopy, and the dashed lines with error regions are Gaussian processing estimates of the interpolated or extrapolated LCs. Relevant upper limits are shown to constrain the early phase of the LCs, displayed as inverted triangles.
Our first Swift/UVOT observation was performed on 2020 July $03(\mathrm{JD}=2459034.4226)$ and provided detections in all the bands. Inspection of the data reveals that it is difficult to assess to what extent the emission is actually from the SN itself, or whether it is diffuse emission from the surroundings. We need to wait for template-subtracted images to get reliable photometry.

\subsubsection{X-rays}

With Swift we also used the on board X-Ray Telescope (XRT; Burrows et al. 2005). We analysed all data with the online tools of the UK Swift team ${ }^{4}$, which use the methods described in

\footnotetext{
4 https://www.swift.ac.uk/user_objects
} 


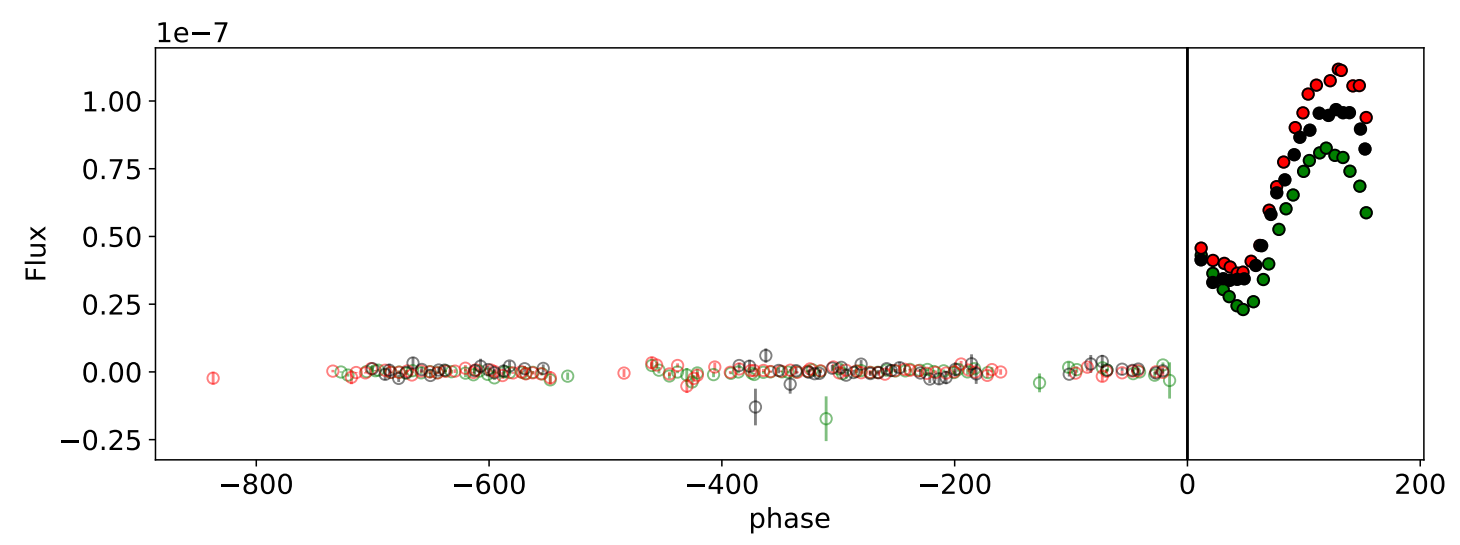

Fig. 3. Pre-explosion images in ZTF for SN 2020faa reveal no precursors in $g$ band (green), $r$ band (red), or $i$ band (black). The flux $f$ is given as a dimensionless ratio and can be converted via $m_{\mathrm{AB}}=-2.5 \log _{10}(f)$. Filled data points are $\gtrsim 5 \sigma$ detections, whereas open points are not significant at the $5 \sigma$ level. The data are binned in seven-day bins.

Evans et al. (2007, 2009), and the software package HEASoft ${ }^{5}$ version 6.26.1 to search for X-ray emission at the location of SN 2020 faa.

Combining the five epochs taken in July 2020 amounts to a total XRT exposure time of $\sim 11000 \mathrm{~s}(3 \mathrm{~h})$, and provides a $3 \sigma$ upper limit of 0.001 count s$^{-1}$ between 0.3 and $10 \mathrm{keV}$. If we assume a power-law spectrum with a photon index of $\Gamma=2$ and a Galactic hydrogen column density of $2.65 \times 10^{20} \mathrm{~cm}^{-2}$ (HI4PI Collaboration 2016) this corresponds to an unabsorbed $0.3-10.0 \mathrm{keV}$ flux of $4 \times 10^{-14} \mathrm{erg} \mathrm{cm}^{-2} \mathrm{~s}^{-1}$. At the luminosity distance of SN 2020 faa this corresponds to a luminosity of less than $L_{\mathrm{X}}=2 \times 10^{41} \mathrm{erg} \mathrm{s}^{-1}(0.3-10 \mathrm{keV})$ at an epoch of $\sim 107 \pm 9$ days rest frame days since discovery.

\subsection{Pre-discovery imaging}

A particular peculiarity for iPTF14hls was the tentative detection of a precursor in images taken in 1954, long before the discovery of the transient. We therefore looked at the P48 imaging of the field of SN 2020faa for some epochs prior to discovery, both by ZTF and by the predecessor PTF. For the PTF images, image subtraction revealed no detection $(5 \sigma)$ for the $65 r$-band images obtained between May 9, 2009, and July 24, 2010. For ZTF, we searched for pre-explosion outbursts in 1538 observations that were obtained in the $g, r$, and $i$ bands in the 2.3 years before the first detection of SN 2020faa. No outbursts are detected when searching unbinned or binned (1- to 90 day bins) LCs following the methods described by Strotjohann et al. (2020); see Fig. 3. The precursor detected prior to iPTF14hls had an apparent $r$ band magnitude of 20.7 (absolute $r$-band magnitude of -15.6) and we can rule out similar outbursts $50 \%$ of the time assuming that an outburst lasts for at least one week.

\subsection{Optical spectroscopy}

Spectroscopic follow-up was conducted with SEDM mounted on the P60 telescope. Further spectra were obtained with the Nordic Optical Telescope (NOT) using the Alhambra Faint Object Spectrograph (ALFOSC). A log of the spectral observations is provided in Table 1, which includes 14 epochs of spectroscopy. SEDM spectra were reduced using the pipeline described by Rigault et al. (2019) and the spectra from La Palma were reduced using standard pipelines. The spectra were finally absolute cali-

\footnotetext{
5 https://heasarc.gsfc.nasa.gov/docs/software/heasoft
}

Table 1. Summary of spectroscopic observations.

\begin{tabular}{lccc}
\hline \hline Object & $\begin{array}{c}\text { Observation date } \\
\text { (YYYY MM DD) }\end{array}$ & $\begin{array}{c}\text { Phase } \\
\text { (Rest frame days) }\end{array}$ & Telescope+Instrument \\
\hline SN 2020faa & 2020 Mar 31 & 6.4 & P60+SEDM \\
SN 2020faa & 2020 Apr 05 & 11.9 & LT+SPRAT \\
SN 2020faa & 2020 Jun 01 & 66.1 & P60+SEDM \\
SN 2020faa & 2020 Jun 21 & 85.2 & P60+SEDM \\
SN 2020faa & 2020 Jul 02 & 96.4 & NOT+ALFOSC \\
SN 2020faa & 2020 Jul 24 & 117.6 & NOT+ALFOSC \\
SN 2020faa & 2020 Jul 26 & 118.8 & P60+SEDM \\
SN 2020faa & 2020 Aug 01 & 124.5 & P60+SEDM \\
SN 2020faa & 2020 Aug 11 & 134.1 & P60+SEDM \\
SN 2020faa & 2020 Aug 15 & 138.6 & NOT+ALFOSC \\
SN 2020faa & 2020 Aug 21 & 143.7 & P60+SEDM \\
SN 2020faa & 2020 Aug 24 & 146.6 & P60+SEDM \\
SN 2020faa & 2020 Aug 30 & 153.0 & NOT+ALFOSC \\
SN 2020faa & 2020 Sep 06 & 159.0 & P60+SEDM \\
\hline
\end{tabular}

brated against the $r$-band magnitudes using the GP interpolated measured magnitudes and then corrected for MW extinction. All spectral data and corresponding information are made available via WISeREP ${ }^{6}$ (Yaron \& Gal-Yam 2012).

\subsection{Host galaxy}

\subsubsection{Photometry}

We retrieved science-ready coadded images from the Galaxy Evolution Explorer (GALEX) general release 6/7 (Martin et al. 2005), the Panoramic Survey Telescope and Rapid Response System (Pan-STARRS, PS1) Data Release 1 (Chambers et al. 2016), the Two Micron All Sky Survey (2MASS; Skrutskie et al. 2006), and pre-processed WISE images (Wright et al. 2010) from the unWISE archive (Lang 2014) ${ }^{7}$. The unWISE images are based on the public WISE data and include images from the ongoing NEOWISE-Reactivation mission R3 (Mainzer et al. 2014; Meisner et al. 2017).

We measured the brightness of the host in a consistent way from the far-ultraviolet to the mid-infrared (i.e. measuring the total flux and preserving the instrinsic galaxy colours) using

\footnotetext{
6 https://wiserep.weizmann.ac.il

7 http://unwise.me
} 
Table 2. Host-galaxy photometry.

\begin{tabular}{lccc}
\hline \hline Survey & Filter & $\begin{array}{c}\text { Wavelength } \\
(\AA)\end{array}$ & $\begin{array}{c}\text { Brightness } \\
(\mathrm{AB} \text { mag })\end{array}$ \\
\hline GALEX & FUV & 1549.0 & $19.30 \pm 0.16$ \\
GALEX & NUV & 2304.7 & $18.68 \pm 0.07$ \\
PS1 & $g$ & 4810.9 & $17.10 \pm 0.03$ \\
PS1 & $r$ & 6156.4 & $16.79 \pm 0.03$ \\
PS1 & $i$ & 7503.7 & $16.56 \pm 0.03$ \\
PS1 & $z$ & 8668.6 & $16.51 \pm 0.03$ \\
PS1 & $y$ & 9613.5 & $16.39 \pm 0.06$ \\
2MASS & $J$ & 12350 & $16.69 \pm 0.19$ \\
2MASS & $H$ & 16620 & $16.23 \pm 0.24$ \\
2MASS & $K$ & 21590 & $16.44 \pm 0.27$ \\
WISE & $W 1$ & 33526 & $16.83 \pm 0.04$ \\
WISE & $W 2$ & 46028 & $17.36 \pm 0.04$ \\
\hline
\end{tabular}

Notes. Magnitudes are not corrected for extinction. The effective wavelengths of the filter response functions are from http://svo2.cab. inta-csic.es/theory/fps/

the Lambda Adaptive Multi-Band Deblending Algorithm in $\mathrm{R}^{8}$ (LAMBDAR; Wright et al. 2016) and the methods described in Schulze et al. (2020). Table 2 gives the measurements in the different bands.

\subsubsection{Spectral energy distribution modelling}

We modelled the spectral energy distribution with the software package prospector version 0.3 (Leja et al. 2017). Prospector uses the Flexible Stellar Population Synthesis (FSPS) code (Conroy et al. 2009) to generate the underlying physical model, and python-fsps (Foreman-Mackey et al. 2014) to interface with FSPS in python. The FSPS code also accounts for the contribution from the diffuse gas (e.g., HII regions) based on the Cloudy models from Byler et al. (2017). We assumed a Chabrier initial mass function (Chabrier 2003) and approximated the star formation history (SFH) by a linearly increasing SFH at early times followed by an exponential decline at late times. The model was attenuated with the Calzetti et al. (2000) model. Finally, we use the dynamic nested sampling package dynesty (Speagle 2020) to sample the posterior probability function.

\subsubsection{Host-galaxy spectrum}

To obtain a spectrum of the host galaxy, we aligned the slit along the host galaxy in our latest NOT observation. In this way we could also extract a spectrum from the region around the SN position in order to measure the host-galaxy emission lines. This was done to carry out an abundance analysis based on the strong line methods.

\section{Analysis and discussion}

\subsection{Light curves}

The $g_{-}^{-}, r-$, and $i$-band LCs of our SN are displayed in Fig. 2. The general behaviour of the LCs was already discussed in Sect. 2.2, and the main characteristic is a slow evolution with an initial decline followed by a late rise over several months. In the figure we have included the most restrictive upper limits $(5 \sigma)$ and the epochs of spectroscopy. The GP interpolation is also shown,

\footnotetext{
8 https://github.com/AngusWright/LAMBDAR
}

which is used for the absolute calibrating of the spectra. For the GP we perform time series forecasting for the joint multi-band fluxes with their corresponding central wavelengths in order to include colour information. Here we use a flat mean function and a stationary kernel Matern 3/2 for the flux form. GP is also used elsewhere in this work to interpolate data, and we then use the flux model from Villar et al. (2019) for the mean function.

In Fig. 4 we show the $g$-, $r$-, and $i$-band LCs in absolute magnitudes together with the LCs of iPTF14hls from S19. The inset at the bottom zooms in on the first 200 days of the evolution of SN 2020faa. The magnitudes in Fig. 4 are in the AB system and have been corrected for distance modulus and MW extinction, and are plotted versus rest frame days past discovery.

The inset shows the remarkable similarity in absolute magnitude and timescale of the two $\mathrm{SNe}$, whereas the full figure might be seen as a prediction for the future evolution of SN $2020 \mathrm{faa}$. No offset was applied to match the absolute magnitudes because they already align quite closely. We also note that no shift was applied in the timescales; we have plotted iPTF14hls since the time of discovery, which supports a similar evolution in this dimension. It is worth noting that the explosion date(or maybe better, time of first light) for iPTF14hls was unconstrained by several months (A17), which made it more difficult to estimate the total radiated energy for that $\mathrm{SN}$, for example. The comparison here makes it likely that iPTF14hls was not discovered very late after all.

Needless to say, the evolution is very different from that of normal SNe Type II, which was already demonstrated by A17 in the comparison of iPTF14hls to SN 1999em (see also Fig. 4). A Type II SN normally stays on a relatively flat plateau for about 100 days, and then quickly plummets to the radioactive decay tail. The rejuvenated long-timescale rise for SN 2020faa argues, as for iPTF14hls, that a different powering mechanism must be at play.

The colour evolution of SN 2020faa is shown in Fig. 5. We plot $g-r$ in the upper panel and $r-i$ in the lower panel, both corrected for MW extinction. In doing this, no interpolation was used. Given the excellent LC sampling we only used data where the pass-band magnitudes were closer in time than 0.1 days. Comparison is made with the colour evolution for iPTF14hls, but this SN was not covered at early phases. There is evidence of similar colours, which argue against significant host extinction. We also compare the colours against a normal Type II SN 2013ej (Valenti et al. 2013), which was selected because it also does not suffer from host-galaxy reddening and has been well monitored in the gri photometric system. These photometric data were obtained via the Open Supernova Catalogue. As shown, SN 2020faa is bluer than the normal Type II SN in $g-r$ colour space, whereas they match closely in $r-i$.

\subsection{Spectra}

The log of spectroscopic observations is provided in Table 1 and the sequence of spectra is shown in Fig. 6. These spectra cover the optical 3200-9500 $\AA$ region. Overall, these are thenspectra of a typical Type II SN. We compare them with spectra from iPTF14hls. We note that the rise of iPTF14hls was not picked up immediately, and therefore the first spectrum of that $\mathrm{SN}$ was only obtained more than 100 days after the first detection. We were faster for SN 2020faa; the first P60 spectrum was obtained six days after discovery, and we can measure the evolution of the expansion velocity from 65 days after discovery.

These velocities are shown in Fig. 7 in comparison to iPTF14hls and SN 1999em values, following the methodology 

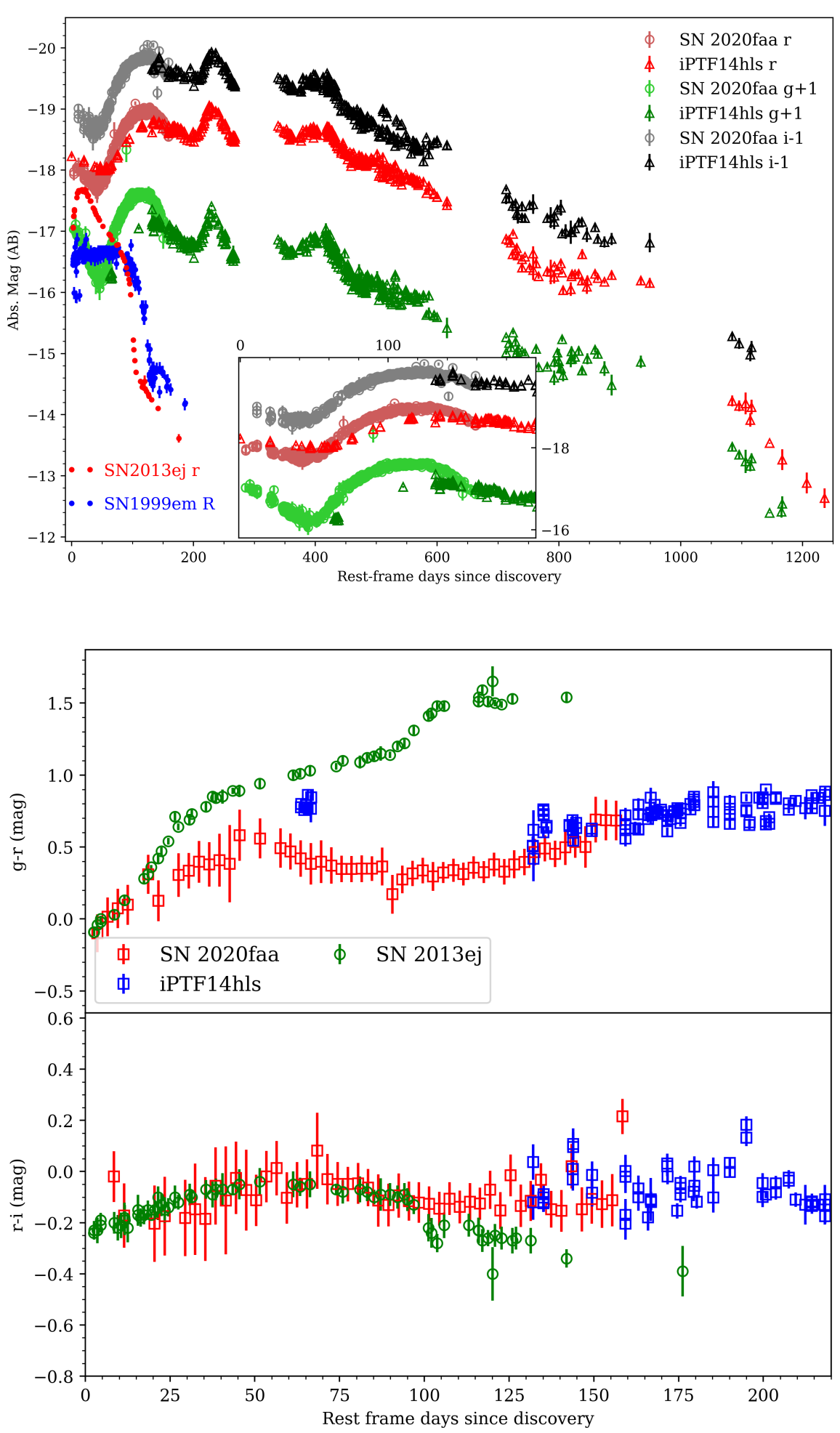

Fig. 4. Absolute magnitudes of SN 2020faa together with the LCs of iPTF14hls, as well as SNe 1999em and 2013ej. No scaling has been applied to match these SNe. The inset shows the early evolution (exactly 200 days) where SN 2020faa demonstrates a striking similarity to the early iPTF14hls LCs.
Fig. 5. Colour evolution of SN 2020faa shown in $g-r$ (upper panel) and $r-i$ (lower panel), binned in three days. The colours have been corrected for MW extinction and are plotted in rest frame days relative to epoch of discovery. For comparison, also shown are the colours for iPTF14hls and for one normal Type II SN 2013ej, whose epochs are also provided in rest frame days since discovery. of A17 (see their Fig. 3). We measured the velocities for SN 2020faa using iraf to fit a Gaussian to the minimum of the absorption lines of the corresponding P Cygni profiles. The difference in rest wavelength between the minimum of the bestfit Gaussian to the line location was translated to an expansion velocity. Uncertainties in the velocities were estimated by a random sampling on the Gaussian minimum 1000 times by $\pm 5 \AA$, as in A17. The time evolution of the velocities measured for $\mathrm{H} \alpha$, $\mathrm{H} \beta$ and for Fe II $\lambda 5169$ closely match those of iPTF14hls at the common epochs, but also extend to earlier phases. The velocities for the comparison are taken from A17. The striking characteristic of the time evolution for iPTF14hls is the very flat velocity evolution. We do not know (yet) if SN 2020faa will follow such a flat evolution, or if iPTF14hls had a faster evolution in the first 100 days.

The velocities for the first two SN 2020faa spectra are not presented in Fig. 7. The first P60 spectrum does not have enough signal, and there is no measurable P Cygni profile in the LT spectrum. However, according to the widths of the emission components of $\mathrm{H} \alpha$ and $\mathrm{H} \beta$, we estimate that the expansion velocities 


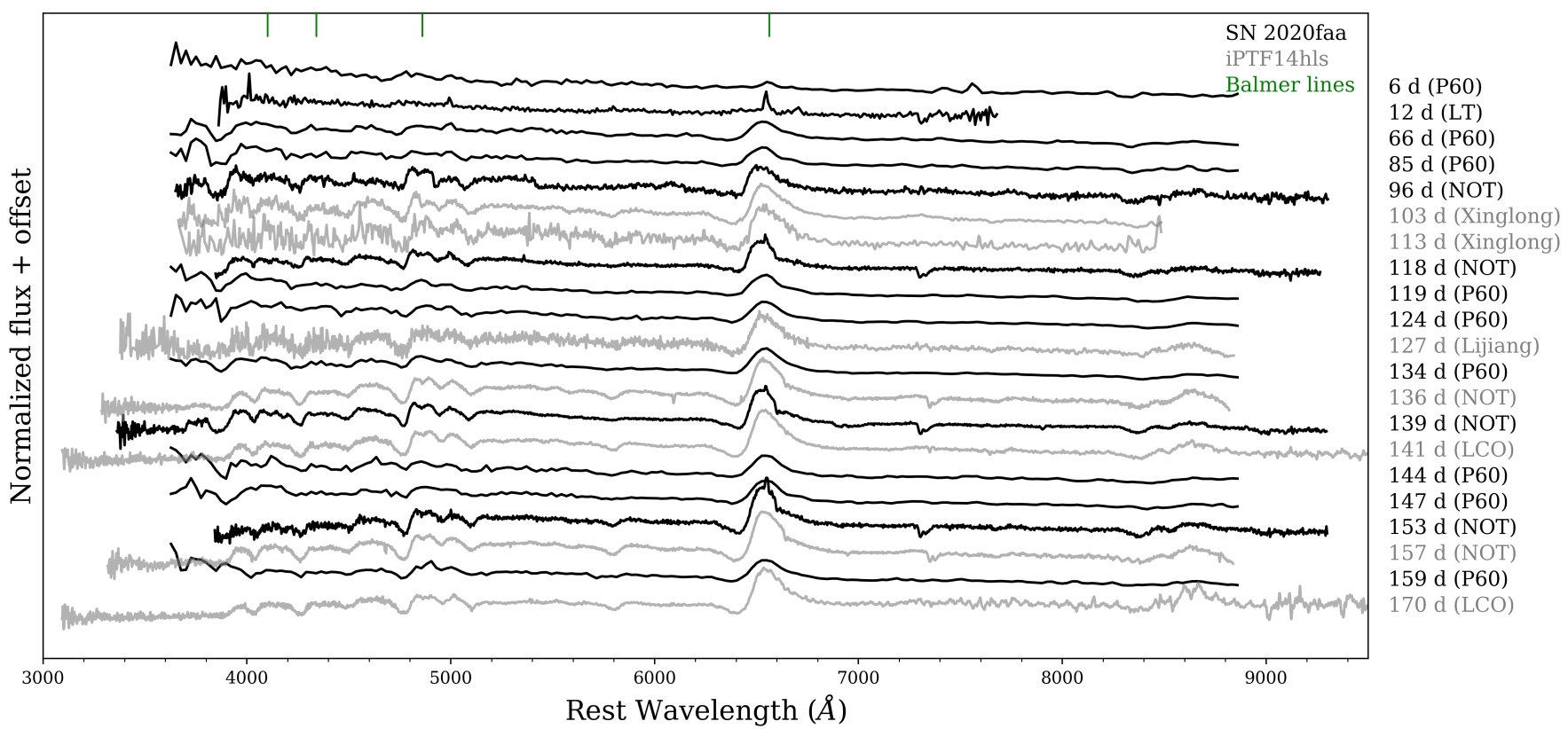

Fig. 6. Sequence of optical spectra for SN 2020faa (in black). The complete log of spectra is provided in Table 1. The epoch of the spectrum is provided to the right. For comparison, also shown are the spectra of iPTF14hls (in grey).

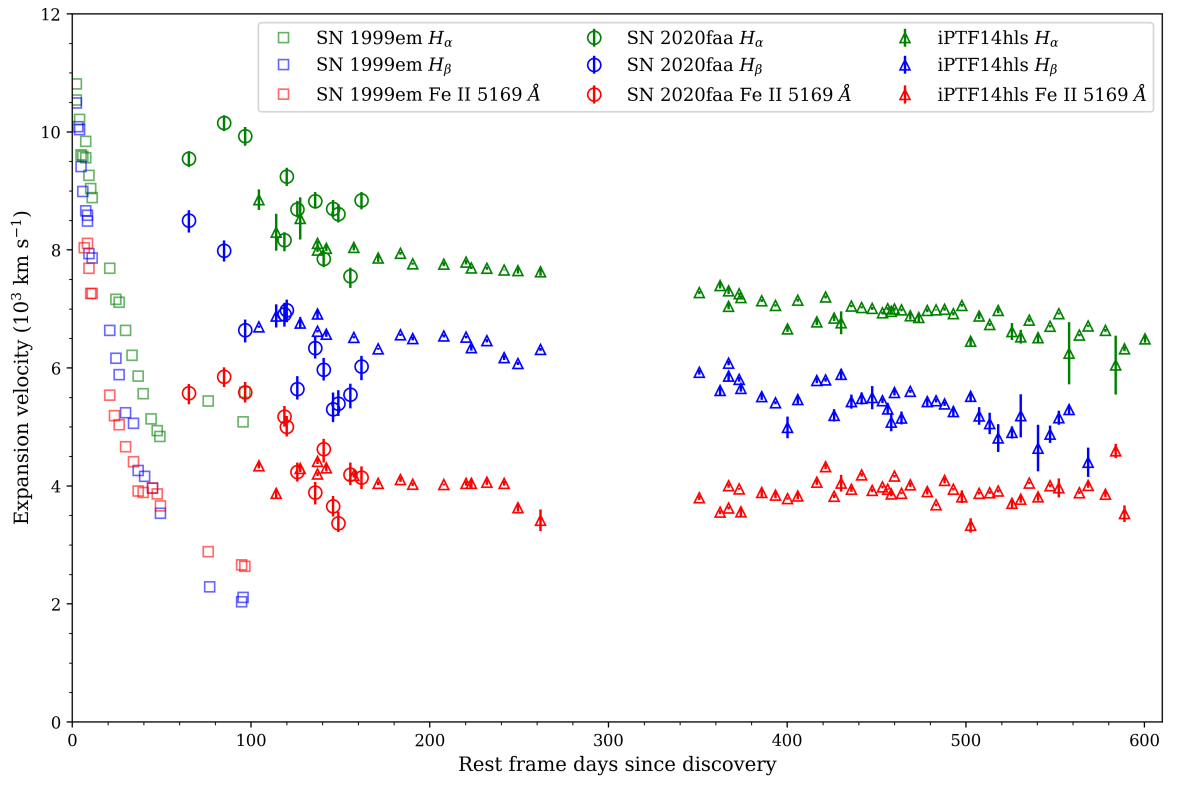

Fig. 7. Velocities estimated from the $\mathrm{P}$ Cygni minima of $\mathrm{H} \alpha$ (green), $\mathrm{H} \beta$ (blue), and Fe II $\lambda 5169$ (red) for the three SNe discussed throughout the paper. The normal Type II SN $1999 \mathrm{em}$ shows a fast decline in expansion velocities, whereas iPTF14hls exhibits virtually constant velocities, with the Fe velocity significantly lower than those estimated from Balmer lines at all epochs. For SN 2020faa we probe intermediate phases and see a slowing down of the photosphere, but with velocities very similar to those demonstrated by iPTF14hls at the common epochs around 150 days. are $\sim 9000 \mathrm{~km} \mathrm{~s}^{-1}$, consistent with those inferred later from the $\mathrm{P}$ Cygni minimum.

A striking characteristic of iPTF14hls was its very slow spectral evolution, with the spectral phase inferred by comparison to typical SNe II (e.g., SN 1999em) being a factor of 6-10 younger than the true phase. To check whether the same is true for $\mathrm{SN}$ 2020faa, we follow A17 and use the spectral comparison code superfit (Howell et al. 2005) to estimate the phase of each spectrum against a library of SN II templates. The results are shown in Fig. 8, with the estimated spectral age plotted versus actual age. The evolution is remarkably flat, and in particular all the spectra taken during the rebrightening phase to date (50-150 days past discovery) match $\mathrm{SN}$ templates with phases 7-30 days past peak light. This is similar to what was seen in A17 (compare their Extended Data Fig. 4), and thus slow spec- tral evolution is another property that iPTF14hls and SN 2020faa have in common.

\subsection{Bolometric light curve}

In order to estimate a total luminosity radiative output, we attempted to construct a bolometric LC. We follow a similar black-body (BB) approximation approach, as done for iPTF14hls by A17, and for the early evolution probed here we have better photometric colour coverage to pursue this.

The result is shown in Fig. 9. The red squares show the luminosity of iPTF14hls (from A17, see their extended data in Fig. 2). There was only enough colour information to fully construct this luminosity for iPTF14hls at later epochs. For SN 2020faa, we can use the gri coverage to estimate the 

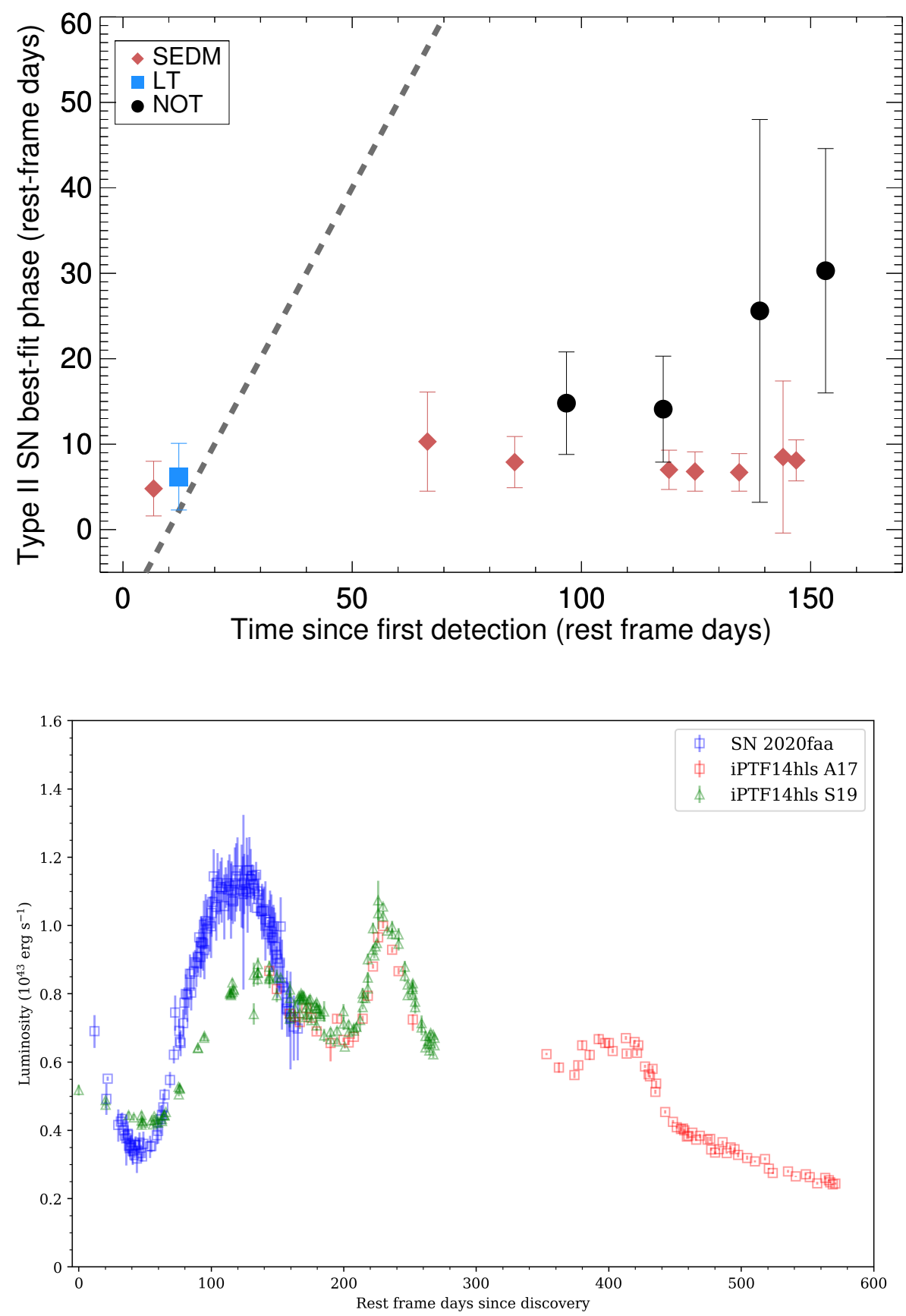

Fig. 8. Phases estimated by comparison to superfit templates are plotted vs rest frame days since the first detection of SN 2020 faa. The overall spectral evolution revealed by these comparisons is very slow, and even at more than 100 days the best matches are with younger Type II SNe. This is similar to what was found by A17 for iPTF14hls, which continued to display slow evolution for $600+$ days. The dashed line shows the expected evolution where the phases would match, assuming a typical time between explosion and peak of ten days.
Fig. 9. Luminosity of SN 2020faa after accounting for MW extinction and distance, and integrating a BB fit to the gri photometry. A similar method was used for iPTF14hls which only had colour data past 150 days, and we can see that the early time emission of SN 2020faa nicely merges with the late time luminosity for iPTF14hls. Shown is the luminosity estimate for iPTF14hls from S19 up to 300 days (in green), assuming a constant bolometric correction at early times. luminosity before this as well, and see that those estimates connect nicely at 150 days post-discovery. Using this data we can estimate a maximum bolometric luminosity for SN 2020faa of $L_{\text {bol }}=1.12 \times 10^{43} \mathrm{erg} \mathrm{s}^{-1}$ at 120.6 rest frame days and a total radiated energy over the first 162.4 rest frame days (until the end of observations presented in this paper, i.e. 2020 September 06) of $E_{\text {rad }}=1.0 \times 10^{50} \mathrm{erg}$. This can be compared to the total radiative output of iPTF14hls, which was $E_{\mathrm{rad}}=3.59 \times 10^{50} \mathrm{erg}$ over 1235 days (S19). In that paper the early bolometric of iPTF14hls was reconstructed, and that comparison is also shown in Fig. 9. Within the uncertainties, they are quite similar; the S19 early bolometric luminosity was estimated from the $r$-band data and a constant bolometric correction. Already the first 160 days of SN 2020faa cannot easily be powered by the mechanism usually responsible for a Type II SN LC-radioactive decay. Using $L=1.45 \times 10^{43} \exp \left(-\frac{t}{\tau_{\mathrm{Co}}}\right)\left(\frac{M_{\mathrm{Ni}}}{M_{\odot}}\right) \mathrm{erg} \mathrm{s}^{-1}$ from Nadyozhin (2003) implies that we would require a solar mass of ${ }^{56} \mathrm{Ni}$ to account for the energy budget. This is already beyond the scope of the traditionally considered neutrino explosion mechanism (e.g., Terreran et al. 2017).

From the BB approximation we also obtain the temperature and the evolution of the $\mathrm{BB}$ radius. The radius evolution was an important clue to the nature of iPTF14hls in A17 (their Fig. 4), and we therefore show a very similar plot in Fig. 10. The radius thus obtained is directly compared to the values for iPTF14hls and SN 1999em. We also include the radius estimated from the spectroscopic velocities, estimated from the P Cygni minima of the Fe II $\lambda 5169$ line.

The figure shows that the BB radius of SN 2020faa at the earliest phases are similar and evolve similarly to those of SN $1999 \mathrm{em}$, and approach the values of the radius for iPTF14hls at 140 days. The $v t$ radius on the other hand are higher for SN 


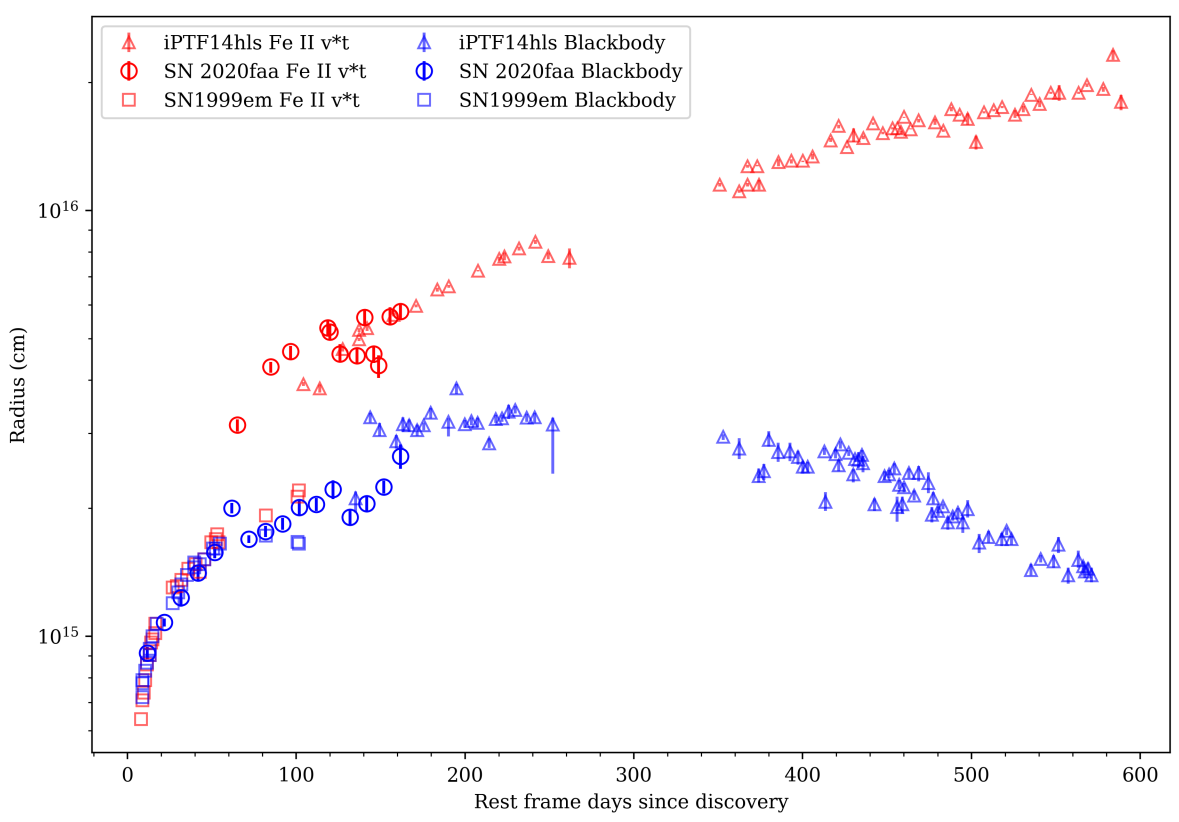

Fig. 10. Evolution of the radius as a function of time for SN 2020faa (binned in 10 days) compared to the extraordinary iPTF14hls and the regular Type II SN 1999em. This figure closely follows the presentation from A17 (their Fig. 4) and shows estimates for the radius evolution from two different methods for the three different SNe. A main theme in A17 was that for iPTF14hls, the radius evolution estimated from the BB approximation and the radius estimated from the spectroscopic velocities were different and diverged with time.

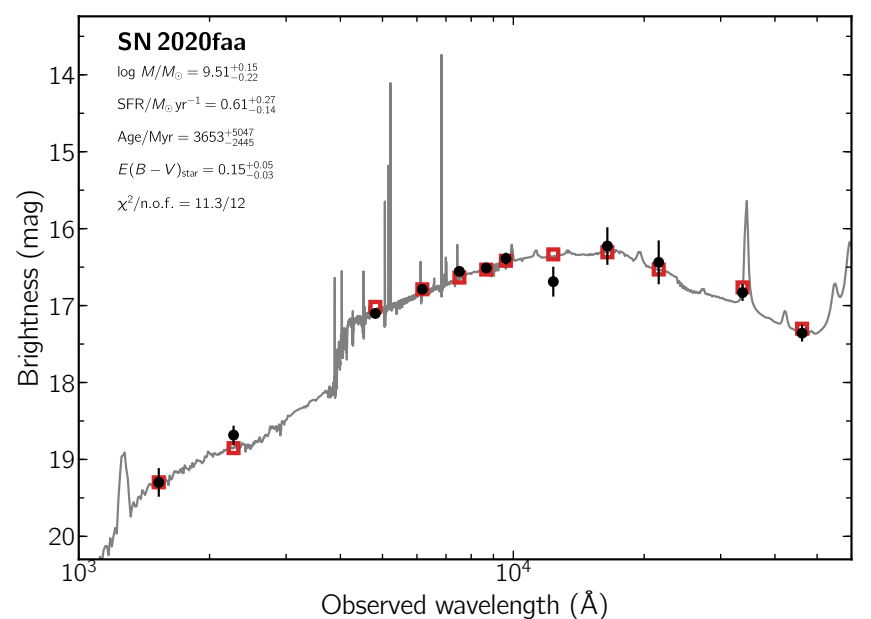

Fig. 11. Spectral energy distribution of the host galaxy of SN 2020 faa from 1000 to $60000 \AA$ (black data points). The solid line displays the best-fitting model of the SED. The red squares represent the modelpredicted magnitudes. The fitting parameters are shown in the upper left corner. The abbreviation "n.o.f." stands for numbers of filters.

2020faa, just as they were for iPTF14hls. We can see that they smoothly connect to the values for iPTF14hls.

\subsection{Host galaxy}

The results of the spectral energy distrubution (SED) modelling (Sect. 2.6.2) of the host galaxy is shown in Fig. 11. We obtain a good fit for a galaxy with a mass of $3.2 \times 10^{9} M_{\odot}$ and a star formation rate of $0.6 M_{\odot}$ per year. This is a relatively regular host galaxy for a Type II SN. In Fig. 12 we compare the host mass with the distribution of host masses for SNe II from the PTF survey from Schulze et al. (2020). As can be seen, the host of SN 2020faa is a regular host galaxy in this respect, and is slightly more massive than the host of iPTF14hls, which is also illustrated in the figure.

Using the emission lines from Sect. 2.6.3, we can adopt the N2 scale of the Pettini \& Pagel (2004) calibration using

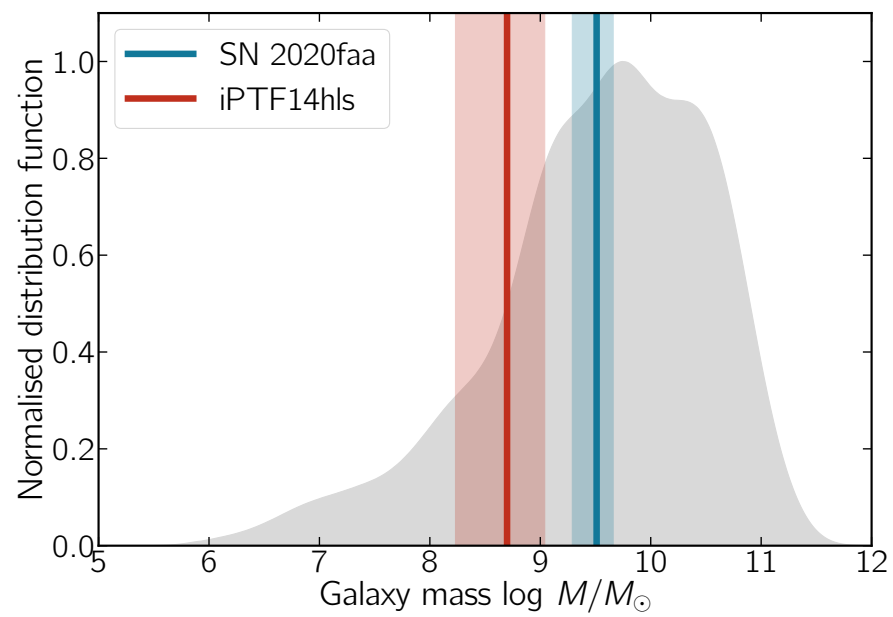

Fig. 12. Host-galaxy mass of SN 2020 faa and iPTF14hls in the context of host galaxies for SNe II from the PTF and iPTF survey (as presented by Schulze et al. 2020).

the flux ratio between [N II] $\lambda 6583$ and $\mathrm{H} \alpha$. We found that $12+\log (\mathrm{O} / \mathrm{H})=8.50 \pm 0.15$. We also employed another metallicity diagnostic of Dopita et al. (2016) using [N II], H $\alpha$ and [S II] lines, in which $12+\log (\mathrm{O} / \mathrm{H})=8.40 \pm 0.10$. Assuming a solar abundance of 8.69 (Asplund et al. 2009), the oxygen abundance of the host galaxy is $0.63_{-0.12}^{+0.26} Z_{\odot}$. Compared to the stellar mass estimate $\left(10^{9.51} M_{\odot}\right)$, our metallicity is consistent with the galaxy mass-metallicity relation (e.g., Andrews \& Martini 2013).

\section{Summary and conclusions}

We have presented SN 2020faa, a young sibling to the spectacular iPTF14hls. The first 150 days of the LC evolution is very different from a normal Type II supernova, and very similar to that of iPTF14hls. We therefore encourage continued monitoring of this transient to explore whether it will evolve in a similar fashion, with LC undulations, longevity, and a slow spectral evolution.

We note that with a declination of $+72^{\circ}$ the source is well placed to be observed around the year from northern observatories. 
From the observations already in hand, we can conclude that just as for iPTF14hls the energy budget is already too high to be driven by a standard radioactivity scenario. The plethora of other powering mechanisms needs to be dusted off again in order to explain the evolution of SN 2020faa. Objects like these challenge the modellers to construct self-consistent models, also for such scenarios as magnetar spin-down and asymmetric CSM-interaction.

The Zwicky Transient Facility will continue operations as ZTFII, with more discoveries in sight. Several community brokers are already processing the data in real time and more activity is foreseen as we come closer to the era of the Vera Rubin telescope. The broker Alerce (Förster et al. 2020) is an example where a combination of computer filtering and human inspection already provides early alerts for infant SNe. We also need to keep an eye on SN LCs that also behave in unusual and interesting ways at later stages. This includes re-brightenings, as for SN 2020faa in this paper, or due to late CSM interaction, as in Sollerman et al. (2020), but could also be rapid declines or undulations, as in iPTF14hls. To date, most of these re-brightenings have been found by human scanners reacting to a 'funny' LC. This will unlikely be the case in the Rubin telescope era. The multitude of machine learning methodologies developed to filter out the interesting targets from the LSST stream will also need to be able to find the unexpected.

Acknowledgements. Based on observations obtained with the Samuel Oschin Telescope 48-inch and the 60-inch Telescope at the Palomar Observatory as part of the Zwicky Transient Facility project. ZTF is supported by the National Science Foundation under Grant No. AST-1440341 and a collaboration including Caltech, IPAC, the Weizmann Institute for Science, the Oskar Klein Center at Stockholm University, the University of Maryland, the University of Washington, Deutsches Elektronen-Synchrotron and Humboldt University, Los Alamos National Laboratories, the TANGO Consortium of Taiwan, the University of Wisconsin at Milwaukee, and Lawrence Berkeley National Laboratories. Operations are conducted by COO, IPAC, and UW. The SED Machine is based upon work supported by the National Science Foundation under Grant No. 1106171. This work was supported by the GROWTH (Kasliwal et al. 2019) project funded by the National Science Foundation under Grant No. 1545949. Partially based on observations made with the Nordic Optical Telescope, operated at the Observatorio del Roque de los Muchachos, La Palma, Spain, of the Instituto de Astrofisica de Canarias. Some of the data presented here were obtained with ALFOSC, which is provided by the Instituto de Astrofisica de Andalucia (IAA) under a joint agreement with the University of Copenhagen and NOTSA. S. Y. and E. K. are funded through the GREAT research environment grant 2016-06012, and E. K. acknowledges support from The Wenner-Gren Foundations. R. L. is supported by a Marie Skłodowska-Curie Individual Fellowship within the Horizon 2020 European Union (EU) Framework Programme for Research and Innovation (H2020-MSCA-IF-2017-794467). T.-W. C. acknowledges the EU Funding under Marie Skłodowska-Curie grant H2020-MSCA-IF-2018-842471. Thanks to David Kaplan for a careful read.

\section{References}

Ahn, C. P., Alexandroff, R., Allende Prieto, C., et al. 2014, ApJS, 211, 17 Andrews, B. H., \& Martini, P. 2013, ApJ, 765, 140

Andrews, J. E., \& Smith, N. 2018, MNRAS, 477, 74

Arcavi, I., Howell, D. A., Kasen, D., et al. 2017, Nature, 551, 210
Asplund, M., Grevesse, N., Sauval, A. J., \& Scott, P. 2009, ARA\&A, 47, 481 Bellm, E. C., Kulkarni, S. R., Graham, M. J., et al. 2019, PASP, 131, 018002 Blagorodnova, N., Neill, J. D., Walters, R., et al. 2018, PASP, 130, 035003 Burrows, D. N., Hill, J. E., Nousek, J. A., et al. 2005, Space Sci. Rev., 120, 165 Byler, N., Dalcanton, J. J., Conroy, C., \& Johnson, B. D. 2017, ApJ, 840, 44 Cardelli, J. A., Clayton, G. C., \& Mathis, J. S. 1989, ApJ, 345, 245 Calzetti, D., Armus, L., Bohlin, R. C., et al. 2000, ApJ, 533, 682 Cenko, S. B., Fox, D. B., Moon, D.-S., et al. 2006, PASP, 118, 1396 Chabrier, G. 2003, PASP, 115, 763

Chambers, K. C., Magnier, E. A., Metcalfe, N., et al. 2016, ArXiv e-prints [arXiv:1612.05560]

Chugai, N. N. 2018, Astron. Lett., 44, 370

Conroy, C., Gunn, J. E., \& White, M. 2009, ApJ, 699, 486

Cook, D. O., Kasliwal, M. M., Van Sistine, A., et al. 2019, ApJ, 880, 7

Dekany, R., Smith, R. M., Riddle, R., et al. 2020, PASP, 132, 038001

Dessart, L. 2018, A\&A, 610, L10

Dopita, M. A., Kewley, L. J., Sutherland, R. S., \& Nicholls, D. C. 2016, Ap\&SS, 361,61

Evans, P. A., Beardmore, A. P., Page, K. L., et al. 2007, A\&A, 469, 379

Evans, P. A., Beardmore, A. P., Page, K. L., et al. 2009, MNRAS, 397, 1177

Foreman-Mackey, D., Sick, J., \& Johnson, B. 2014, https://doi.org/10. 5281/zenodo. 12157

Förster, F., Cabrera-Vives, G., Castillo-Navarrete, E., et al. 2020, AAS J. submitted, [arXiv:2008.03303]

Fremling, C., Sollerman, J., Taddia, F., et al. 2016, A\&A, 593, A68 Gehrels, N., Chincarini, G., Giommi, P., et al. 2004, ApJ, 611, 1005 Graham, M. J., Kulkarni, S. R., Bellm, E. C., et al. 2019, PASP, 131, 078001

HI4PI Collaboration (Ben Bekhti, N., et al.) 2016, A\&A, 594, A116 Howell, D. A., Sullivan, M., Perrett, K., et al. 2005, ApJ, 634, 1190

Kasliwal, M. M., Cannella, C., Bagdasaryan, A., et al. 2019, PASP, 131, 038003 Lang, D. 2014, AJ, 147, 108

Leja, J., Johnson, B. D., Conroy, C., van Dokkum, P. G., \& Byler, N. 2017, ApJ, 837,170

Mainzer, A., Bauer, J., Cutri, R. M., et al. 2014, ApJ, 792, 30

Martin, D. C., Fanson, J., Schiminovich, D., et al. 2005, ApJ, 619, L1

Masci, F. J., Laher, R. R., Rusholme, B., et al. 2019, PASP, 131, 018003

Meisner, A. M., Lang, D., \& Schlegel, D. J. 2017, AJ, 153, 38

Moriya, T. J., Mazzali, P. A., \& Pian, E. 2019, MNRAS, 491, 1384

Nadyozhin, D. K. 2003, MNRAS, 346, 97

Perley, D. A., Taggart, K., Dahiwale, A., \& Fremling, C. 2020, Transient Name Server Classification Report, No. 2020-987, 1

Pettini, M., \& Pagel, B. E. J. 2004, MNRAS, 348, L59

Rigault, M., Neill, J. D., Blagorodnova, N., et al. 2019, A\&A, 627, A115

Roming, P. W. A., Kennedy, T. E., Mason, K. O., et al. 2005, Space Sci. Rev., 120,95

Schulze, S., Yaron, O., Sollerman, J., et al. 2020, ArXiv e-prints [arXiv:2008.05988]

Skrutskie, M. F., Cutri, R. M., Stiening, R., et al. 2006, AJ, 131, 1163

Soker, N., \& Gilkis, A. 2017, MNRAS, 475, 1198

Sollerman, J., Taddia, F., Arcavi, I., et al. 2019, A\&A, 621, A30

Sollerman, J., Fransson, C., Barbarino, C., et al. 2020, A\&A, 643, A79

Speagle, J. S. 2020, MNRAS, 493, 3132

Strotjohann, N. L., Ofek, E. O., Gal-Yam, A., et al. 2020, ArXiv e-prints [arXiv:2010.11196]

Terreran, G., Pumo, M. L., Chen, T. W., et al. 2017, Nat. Astron., 1, 713

Tonry, J., Denneau, L., Heinze, A., et al. 2020, Transient Name Server Discovery Report, No. 2020-869, 1

Valenti, S., Sand, D., Pastorello, A., et al. 2013, MNRAS, 438, L101

Villar, V. A., Berger, E., Miller, G., et al. 2019, ApJ, 884, 83

Wang, L. J., Wang, X. F., Wang, S. Q., et al. 2018, ApJ, 865, 95

Woosley, S. E. 2018, ApJ, 863, 105

Wright, E. L., Eisenhardt, P. R. M., Mainzer, A. K., et al. 2010, AJ, 140, 1868

Wright, A. H., Robotham, A. S. G., Bourne, N., et al. 2016, MNRAS, 460, 765

Yaron, O., \& Gal-Yam, A. 2012, PASP, 124, 668 